\title{
Ardeth
}

A magazine on the power of the project

$7 \mid 2020$

Europe

\section{Progettare l'integrazione.}

Lo spazio dell'incontro e dello scambio nel futuro delle città multiculturali

Design the Integration. The Space of Encounter and Exchange in the Future of Multicultural Cities.

Manuela Raitano, Angela Fiorelli e Gloria Riggi

(CpenEdition

Journals

Edizione digitale

URL: https://journals.openedition.org/ardeth/1848

ISSN: 2611-934X

Editore

Rosenberg \& Sellier

Edizione cartacea

Data di pubblicazione: 1 décembre 2020

Paginazione: 107-125

ISSN: 2532-6457

Notizia bibliografica digitale

Manuela Raitano, Angela Fiorelli e Gloria Riggi, «Progettare l'integrazione.», Ardeth [Online], 7 | 2020,

online dal 01 juin 2021, consultato il 25 juin 2021. URL: http://journals.openedition.org/ardeth/1848

CC BY-NC-ND 4.0 


\section{Progettare l'integrazione. Lo spazio dell'incontro e dello scambio nel futuro delle città multiculturali}

Manuela Raitano (1); Angela Fiorelli (2); Gloria Riggi (3)

Abstract / Design the Integration. The Space of Encounter and Exchange in the Future of Multicultural Cities. The phenomenon of migratory flows constitutes an emergency in Europe. At the moment, architects are not directly involved in technical and institutional boards, while we know that the quality of urban spaces is crucial, since it is starting from space - and its conformation - that one can manage the interrelations between the individuals. Our cities must be ready to absorb the impact of migrants, accepting multiculturalism as a resource and not a threat. In addition to the places for the first aid, it is now essential to introduce also new architectural models, aimed at assistance and integration of migrants in the host communities. Starting from the assumption that the "right to the city" must be universally extended - and taking the Termini station area in Rome as a casestudy - we tried to prefigure new models for multifunctional buildings in which cultural pluralism can be developed, respecting and protecting each person's identity.

Affiliatio

(1) (2) (3) Sapienza Università di Roma, DiAP - Dipartimento di Architettura e Progetto

Contacts:

(1) manuela [dot] raitano [at] uniroma1 [dot] it (2) angela [dot] fiorelli [at] unir ma1 [dot] it (3) gloria [dot] riggi [at] uniroma1 [dot] it

Received:

20 April 2020

Accepted:

21 November 2020

DOI:

10.17454/ARDETH07.07

ARDETH \#07 
L’antica そcvía - il diritto all'ospitalità che per secoli era stato garantito dalle leggi dell'antico

Mediterraneo - non sembra oggi

costituire elemento di riconoscimento comune per l’identità europea.
I flussi migratori in Europa e il diritto alla città Alcune recenti risoluzioni degli stati membri d'Europa sembrano assecondare un approccio divisivo e diffidente nei confronti dell'immigrato. L'impatto dei grandi flussi migratori ha infatti generato non pochi conflitti sociali all'interno dei paesi comunitari, alterando i già precari equilibri tra l'area mediterranea e gli stati del Nord e Centro Europa. Ne è conseguito, inevitabile, un diffuso sentimento di ostilità verso quanti non detengono il cosiddetto "diritto di cittadinanza”, acuitosi a seguito della crisi economica mondiale del 2008 e degli attacchi terroristici di matrice fondamentalista. In questo quadro l'antica $\xi \varepsilon v i ́ a-i l$ diritto all'ospitalità che per secoli era stato garantito dalle leggi dell'antico Mediterraneo - non sembra oggi costituire elemento di riconoscimento comune per l'identità europea. I migranti approdano così in un continente culturalmente impreparato ad accoglierli e a pianificarne la permanenza.

È qui in primis necessario specificare che con il termine "migranti" siamo costretti ad accomunare sotto un'unica parola una moltitudine di differenti persone e di esperienze: individui con status sociali, economici e culturali diversi, che vanno dai cosiddetti clandestini, non legalmente riconosciuti, fino ai rifugiati politici; o dagli extracomunitari in transito fino ai residenti in forma stabile, con regolare permesso di soggiorno. Tutti costoro rappresentano, nel loro insieme, un gruppo sociale multiforme ed eterogeneo di soggetti deboli, a cui le politiche dei vari stati dovrebbero rispondere in termini di integrazione, e non solo di prima accoglienza.

"Integrazione" è tuttavia parola che va anch'essa chiarita: da un lato, infatti, si può affermare che la contaminazione culturale sia una necessità primaria per una società coesa e multietnica; dall'altro bisogna ricordare che tale obiettivo va comunque raggiunto a partire dalla tutela delle singole identità di provenienza. Pertanto, l'integrazione non deve intendersi come l'attuarsi di un processo coatto di cancellazione delle differenze, quanto piuttosto come l'innesco di un processo bilaterale di scambio. $\mathrm{E}$ in tale processo l'architettura può e deve giocare la sua parte. Come scrive infatti Rosario Pavia, «non solo le città non sono accoglienti, ma il pianeta stesso diventa sempre più invivibile», al punto che «per molti versi l'accoglienza 
pone una questione etica che investe pienamente la cultura dell'architettura moderna» (Pavia, 2016). In questa prospettiva diventa allora importante chiedersi se le città europee, e segnatamente quelle italiane, siano attrezzate per accogliere. Per quanto infatti si stia diffondendo l'idea che modelli multietnici siano più facilmente realizzabili in realtà medio-piccole come i centri minori (si pensi al caso di Rosarno in Calabria), non c'è dubbio che lo spazio tradizionalmente cosmopolita delle grandi città sia ancora da ritenersi il luogo deputato all'attuazione dei processi di integrazione. Le città sono sì purtroppo teatro di conflitti, vi proliferano fenomeni di intolleranza e razzismo, ma possono anche offrire opportunità di riconoscimento sociale a gruppi di individui differenti per provenienza e modelli di vita. Al punto che quasi potremmo affermare che l'immigrazione sia oggi «un fatto urbano totale, perché coinvolge ogni aspetto, ogni dimensione e ogni rappresentazione dell'assetto sociale, spaziale, politico, culturale e religioso delle città»(Urban@it, 2016).

A partire da questo assunto, la questione centrale diviene quella del "diritto alla città"; diritto che dovrà valere, in Europa come altrove, per qualunque individuo, comunitario e non, residente o "in transito" (Criconia, 2019). Va tuttavia considerato che tale diritto è in sé contraddittorio, poiché l'idea di città "per tutti" entra spesso in conflitto, nell'immaginario collettivo, con la percezione della sicurezza "del singolo" (Agostini et al., 2017), riverberando così dall’opinione pubblica alla risposta politica. Se da un lato, dunque, si dovranno garantire apertura e solidarietà, dall'altro si dovranno anche garantire, nelle città contemporanee, protezione e controllo (Bauman, 2016), ma scardinando il luogo comune che vede tale garanzia inficiata dalla presenza delle minoranze multietniche.

Nei termini della costruzione dello spazio urbano, i due concetti antitetici di apertura e controllo, per quanto schematici, ci aiutano a rappresentare i poli opposti della questione: l'apertura si progetta infatti per via di spazi permeabili, che attraversino tutte le gradazioni che vanno dal semi-pubblico al semi-privato, che invoglino alla scoperta ma che, allo stesso tempo, si comprendano intuitivamente. Il controllo al contrario richiede che sia chiara la distinzione tra il pubblico e il privato, tra il dentro e il fuori.
Diventa allora
importante
chiedersi se le
città europee, e
segnatamente
quelle italiane,
siano attrezzate per
accogliere. 


\section{Sarà dunque} necessario operare per attrezzare spazi urbani condivisi $m a$ sicuri, plurali $m a$ identitari.

\section{I luoghi}

istituzionali

dell'accoglienza,

nella grande

maggioranza

dei casi, sono

sistemazioni

di fortuna che

occupano edifici in

disuso o strutture

removibili e

temporanee.
Nel primo caso abbiamo la sovrapposizione dei flussi come valore, nel secondo la loro distinzione; e ancora: nel primo caso ci muoviamo nel campo della cultura dell'et-et, cioè nel campo della mediazione, nel secondo invece in quella dell'aut-aut, che prevede la separazione e la distinguibilità degli enti.

In questa cornice non univoca, definita da un quadro valoriale difficilmente riconducibile alla pacificazione, sarà dunque necessario operare per attrezzare spazi urbani condivisi ma sicuri, plurali ma identitari. Ed è sotto questo punto di vista che le competenze specifiche dell'architetto potranno rivelarsi dirimenti. Per questa ragione stupisce constatare come, al momento attuale, l'architettura non sia chiamata direttamente in causa nei tavoli tecnico-istituzionali, da un lato perché non viene capito come la costruzione dello spazio sia parte della soluzione del problema, dall'altro perché ancora scontiamo l'identificazione pubblica con una figura professionale sovrastrutturale, che si occupa di puro godimento estetico, identificazione di cui il sentire postmoderno si è reso evidentemente complice. Mentre, al contrario, il tema dello spazio per l'inclusione può rappresentare una vera occasione per rimettere la nostra disciplina, se non al centro del dibattito, almeno a lato dei tavoli istituzionali di livello europeo.

La presenza dei migranti nelle città impatterà infatti sul disegno degli spazi collettivi, sugli edifici scolastici, sulla qualità dei servizi ecc.; va pertanto predisposto uno scenario virtuoso in cui si auspica di superare l'ottica emergenziale, per lavorare in prospettiva a "progettare l'integrazione".

\section{I luoghi dell'accoglienza in Italia}

Entrando nello specifico, nel nostro paese i luoghi istituzionali dell'accoglienza, nella grande maggioranza dei casi, sono sistemazioni di fortuna che occupano edifici in disuso o strutture removibili e temporanee. Manca una programmazione, e non si è consolidata una ricerca tipologica sul tema.

La normativa, in particolare, non aiuta a definire nuovi modelli: essa tratta di strutture per "l'accoglienza", ma mai di strutture per "l'integrazione"; non definisce inoltre tipologie di ambienti né programmi funzionali, né tenta di stabilire minimi inderogabili né princìpi capaci di normare le relazioni tra i luoghi deputati ai migranti e la città stessa. Dal punto di vista 


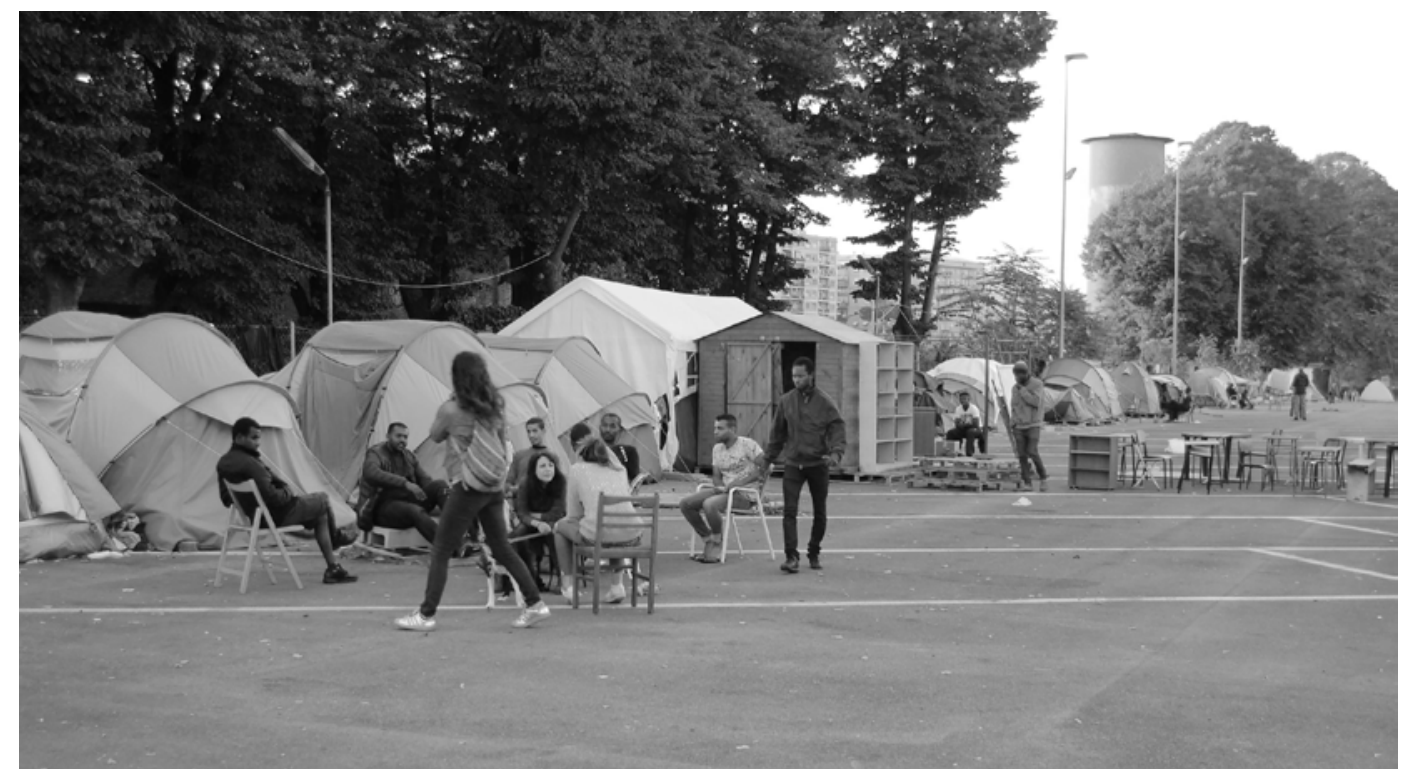

normativo vengono anzi incentivate, in Italia, occupazioni provvisorie di edifici dismessi, ovvero strategie di adattamento che, se da un lato risolvono l'emergenza, dall'altro non riescono a definire soluzioni ad hoc. Sono perciò riutilizzati, in molti casi, alberghi abbandonati, ex caserme ecc., senza tenere in conto le possibili dinamiche di emarginazione e l'innescarsi del cosiddetto "effetto-ghetto", laddove il termine è qui usato non nel significato (oggi riconsiderato) di struttura organizzata e comunitaria, ma nell'accezione comune di luogo della segregazione: "ghetto significa impossibilità di creare una comunità», afferma infatti Zygmunt Bauman (2001), riassumendo le parole di Loic Wacquant.

A lato di quanto detto finora, ancor meno viene valutato l'impatto che i migranti producono nel tessuto spaziale e morfologico delle nostre città. Conseguenza di ciò è che, a fronte di una mancanza di strutture dedicate, si innescano meccanismi di autoregolamentazione da parte dei migranti stessi, che allestiscono i propri spazi in forma autonoma, "espropriando" parti di città. Questi fenomeni non sono confinati nelle sole periferie, ma coinvolgono aree urbane anche non marginali: il quartiere Flaminio a Roma ne è l'esempio, con Piazza Mancini che si trasforma, ogni fine settimana, nel punto di incontro delle comunità latine
Fig. 1 - II presidio di piazzale Maslax a Roma, gestito da Baobab Experience, sgomberato nel novembre 2018. Foto di G. Dal Bianco.

\section{Vengono incentivate, occupazioni provvisorie di edifici dismessi, ovvero strategie di adattamento che, non riescono a definire soluzioni ad hoc.}


1 - Secondo la Road-

map italiana del settembre 2015, redatta dal Ministero dell'Interno, dopo l'identificaz one negli hotspot, $i$ migranti richiedenti asilo vengono trasferiti nei vari regional hub presenti sul territorio nazionale, mentre gli irregolari sono trasferiti nei Centri di Identificazione ed Espulsione. locali; in questi luoghi l'utilizzo spontaneo da parte delle comunità straniere, di per sé lecito, entra in conflitto con le regole di fruizione ordinaria; va da sé che l'assenza di un progetto tanto politico quanto urbano si traduce, nella percezione dei residenti, nella forma di un'appropriazione indebita che induce reazioni di diffidenza e intolleranza.

Numerosi sono, a Roma, gli esempi: tra questi è noto il presidio di piazzale Maslax, situato in un'area prossima alla stazione Tiburtina, gestito dall'associazione Baobab e sgomberato infine nel 2018; questi insediamenti, che pure rispondono a un criterio di necessità, non aiutano purtroppo a favorire la pacificazione sociale, essendo spesso avvertiti come atti di "spoliazione” illecita. Al contrario, attraverso lo strumento del progetto, si potrebbero predisporre tali spazi in modo da promuoverne l'uso condiviso tra extracomunitari e residenti. Locale e globale potrebbero così intrecciarsi e interagire in forme sconosciute nelle epoche precedenti, producendo per tutti, nelle città, inediti luoghi-sosta di radicamenti dinamici e mobilità multiformi.

Con riferimento all'assetto normativo, l'accoglienza in Italia è regolata dal D.Lgs n. 142 del 2015, che si basa sulle disposizioni proposte dalla Conferenza Unificata Stato Regioni del 10 luglio 2014, in ottemperanza alle Direttive europee e, successivamente, anche ai D.Lgs 13/2017 e 113/2018; tutte queste disposizioni, unitamente al recente D.L. del 5 ottobre 2020, definiscono alcuni importanti aspetti in materia di immigrazione, protezione internazionale, libera circolazione nell'Unione Europea e riconoscimento del singolo ${ }^{1}$.

Il sistema di accoglienza è articolato, in sintesi, in tre momenti: una fase "zero", che si svolge in strutture denominate hotspot, dedicata al soccorso e alla prima identificazione; una fase "uno" che riguarda centri in cui si attuano procedure di supporto psicologico, assistenza medico-legale e attività quotidiane di sostentamento; infine, una fase detta di "seconda accoglienza”, in cui le strutture di riferimento sono quelle del sistema SPRAR (Sistema di Protezione per Richiedenti Asilo e Rifugiati). Questo sistema di accoglienza viene poi affiancato e supportato da altre strutture, in particolare i CAS (Centri di Accoglienza Straordinaria), i Centri di Accoglienza per Richiedenti Asilo (CARA), i CIE (Centro di identificazione ed espulsione) e i CPR 
(Centro di permanenza per i rimpatri). Molto spesso, si verifica che la maggioranza dei migranti venga accolta nelle strutture straordinarie (i CAS) piuttosto che in quelle ordinarie (gli SPRAR), poiché le prime funzionano con minori obblighi di controllo e di rendicontazione (Firouzi, 2018).

Per dare maggior ordine all'apparato sopra descritto, il Ministero dell'Interno ha inoltre redatto una roadmap dell'accoglienza, istituendo strutture di scala maggiore: i regional hubs, grandi centri a livello regionale e/o interregionale dove fare un primo screening dei migranti che abbiano formalmente espresso la volontà di richiedere protezione. In sostanza, essi rappresentano una sorta di centro di smistamento dove le persone dovrebbero risiedere per poco tempo (dai 7 ai 30 giorni) per realizzare le operazioni di identificazione e formalizzazione della domanda di protezione ed essere poi trasferiti nei centri di seconda accoglienza, vale a dire nelle strutture della rete SPRAR e CAS. All'interno della struttura legislativa, il tema dello spazio architettonico è tuttavia il grande assente: nella normativa in materia sono infatti indicati i procedimenti da seguire e la definizione giuridica delle strutture deputate, ma non è riportato come fisicamente possa essere uno spazio per l'accoglienza. Le strutture non sono suddivise in base ai loro principi formativi e funzionali, ma in base al soggetto giuridico da ospitare (Urban@it, 2016), né la scelta della localizzazione di un centro all'interno di un territorio è considerata strategica, ai fini della riuscita dei processi di integrazione. Sotto questo aspetto la ricerca accademica può contribuire offrendo competenze specifiche, capaci di prefigurare strutture innovative all'interno delle comunità ospitanti.

Verso un modello di architettura per l'accoglienza: il caso di Roma Termini

Il lavoro che qui presentiamo intende soffermarsi sulla necessità di un'“architettura per l'inclusione”, in un contesto, quello del quartiere Esquilino a Roma, dove è forte la presenza di una componente extracomunitaria che richiede con urgenza una nuova visione sistemica, di programma e di azione. La tesi di fondo, su cui è articolato il progetto di ricerca ${ }^{2}$, è che gli spazi dedicati ai migranti possano (e debbano) essere pensati non solo per offrire servizi primari alle
2 - Nel testo si fa riferimento, nello specifico, alla rice ca di Ateneo “Roma Termini. La piazza e la città, tra patrimonio e infrastruttura" in corso di svolgimento presso il Dipartimento di Architettura e Progetto, Sapienza Università di Roma, resp. M. Raitano, con A. Fiorelli, G. Riggi et al.

\section{All'interno della struttura legislativa, il tema dello spazio architettonico è tuttavia il grande assente.}


Fig. 2 - Roma, via

Gioberti. Sulla

sinistra l'isolato

parzialmente demo-

lito, prospiciente la

stazione, nel quale si

prevede di ubicare la

"Casa delle Culture".

Foto di S. Leoni.
Si consideri che attualmente non esistono, nella città di Roma, strutture centralizzate in grado di fornire tutte insieme i servizi essenziali di cui il migrante necessita nei primi mesi di permanenza nel paese a lui straniero. minoranze, ma anche per offrire servizi aggiuntivi ai nativi locali. Attraverso la lente disciplinare viene così indagata la possibilità di un impegno diretto, di larga scala, dell'architetto, nella costruzione di una città che, già multietnica, dovrà divenire inclusiva e interculturale. Lo scenario è, come già detto, la città di Roma, ma il progetto viene impostato secondo un approccio strategico, virtualmente trasferibile altrove. Nello specifico, tale ricerca intende porsi a valle del processo sopra descritto, che regolamenta la cosiddetta "seconda accoglienza". Non interessa perciò indagare nuovi modelli per i centri del sistema SPRAR, bensì nuovi spazi per rendere più facile - e integrata - la vita del migrante, una volta ch'egli è uscito dalla fase dell'accoglienza di base, quando ormai è inserito nel corpo fisico di una grande città che non conosce e che non lo riconosce.

Si consideri che attualmente non esistono, nella città di Roma, strutture centralizzate in grado di fornire tutte insieme i servizi essenziali di cui il migrante necessita nei primi mesi di permanenza nel paese a lui straniero: egli vaga, pertanto, da un giardino pubblico alla sede di una onlus, dalla mensa al centro di volontariato e via così. In un certo senso, continua a migrare nelle città, non superando realmente quella condizione coatta in cui viveva prima dell'arrivo. L'obiettivo primario della ricerca è stato quindi, innanzitutto, capire come provvedere a fornirgli i servizi essenziali, evitando di disperderli sul territorio, ma concentrandoli in strutture ad essi deputate. Si è trattato pertanto di immaginare strutture complesse, che potrebbero essere gestite su base volontaria, come del resto già accade per gli $h u b$ la cui gestione, a oggi, vede in Italia, in prima linea, le associazioni umanitarie e le onlus.

Il tema della localizzazione è altrettanto centrale, in quanto la scelta delle aree dove insediare questo genere di servizi urbani andrebbe coordinata su scala nazionale, sulla base di dati incrociati. In particolare, sappiamo che nelle grandi città i flussi migratori investono principalmente le stazioni, e in particolare quelle di Milano Centrale, Roma Termini, Roma Tiburtina, Ventimiglia e Bolzano. Per questa ragione, il gruppo Ferrovie dello Stato, dal 2015, ha messo a disposizione diverse strutture dismesse adibite a centri di prima accoglienza, tutte limitrofe alle stazioni: il primo è stato l'hub "Migranti" della Stazione Centrale 


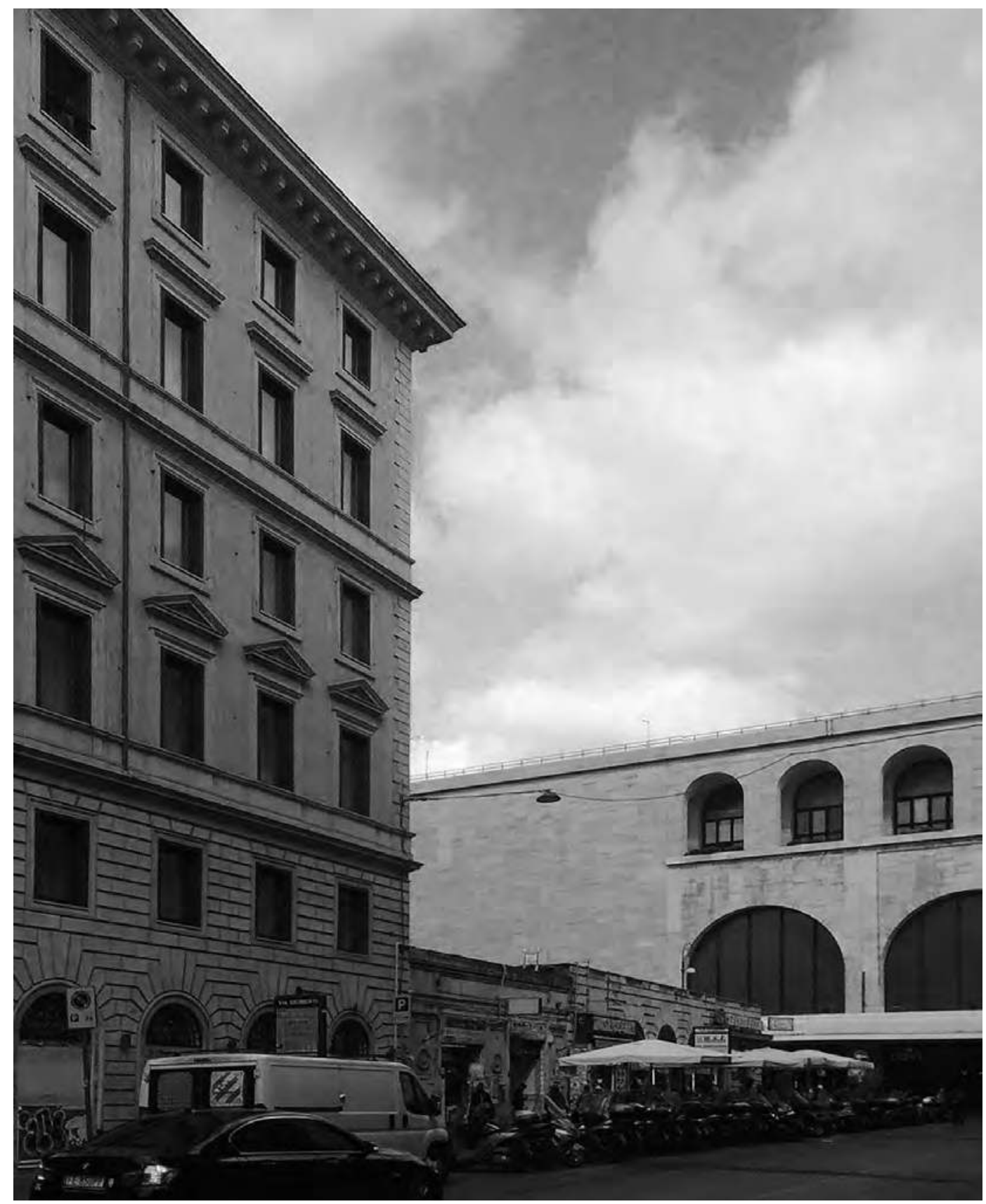


3 - II centro potrà accogliere fino a 150 persone per notte, in $\mathbf{5 0}$ camere, e disporrà di un presidio sanitario, ma ancora non è stato realizzato (aggiornamento febbraio 2020). di Milano (1500 mq, in via Sammartini) gestito dalla Fondazione Progetto Arca, che è stato attivo dal 2015 al 2018 e successivamente trasformato in un presidio per persone senza fissa dimora. Questo modello sarà forse riproposto anche a Roma, dove Ferrovie dello Stato ha in programma di concedere al Comune, in comodato gratuito, un ex ferrotel di $1100 \mathrm{mq}$, posto di fronte alla stazione Tiburtina ${ }^{3}$. In accordo con queste politiche di sviluppo, si è scelto pertanto di focalizzare il progetto sulle aree ferroviarie: i "porti” di approdo alle grandi città sono stati infatti ritenuti luoghi vitali, capaci di assorbire l'impatto della multiculturalità, valorizzandone la presenza.

Facciamo a questo punto un breve riepilogo dello stato dell'arte: in primis il sistema normativo, come si è scritto, non ci aiuta a definire le qualità architettoniche di queste strutture; in secundis molti servizi primari cui si rivolgono questi nostri concittadini "irregolari" sono attualmente ubicati in differenti parti della città, spesso in luoghi periferici dove si incontrano maggiori difficoltà di integrazione sociale; in ultimo, molte delle strutture provvisionali in uso non sono in grado di svolgere più di una funzione alla volta. Tutto questo comporta, come si diceva, il continuo rimpallo di questi soggetti deboli da un capo all'altro della città per mangiare, per ricevere assistenza legale, per socializzare, per dormire ecc. Accanto a ciò si generano, attraverso una miriade di insediamenti informali, pratiche adattive di trasformazione urbana per nulla virtuose.

Tenuto conto di tali condizioni al contorno, il gruppo di ricerca ha valutato l'ipotesi di studiare in astratto un nuovo tipo edilizio, e cioè un contenitore polifunzionale, posto in prossimità delle grandi stazioni (italiane ed europee) nel quale accentrare le funzioni dedicate all'assistenza, mescolandole con quelle legate all'integrazione e quindi alla dotazione di quegli strumenti, materiali e immateriali, che favoriscano l'autonomia degli individui nel paese ospitante. Segnatamente, si sono sviluppate alcune proposte riguardanti il principale nodo su ferro della città di Roma, costituito dalla Stazione Termini e dall'antistante piazza dei Cinquecento.

Il caso di Roma Termini viene da noi assunto quale palinsesto fisico per sperimentare l'idea di un nuovo tipo di "edificio-mondo", dalla forte valenza urbana, capace di ribaltare la percezione del fenomeno soprattutto dal punto di vista dei cittadini residenti, che 

6. $* 140$.

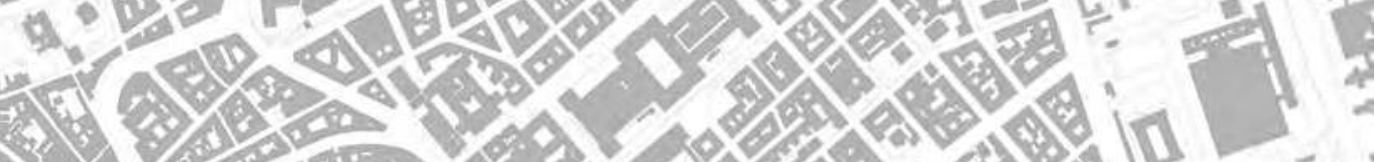

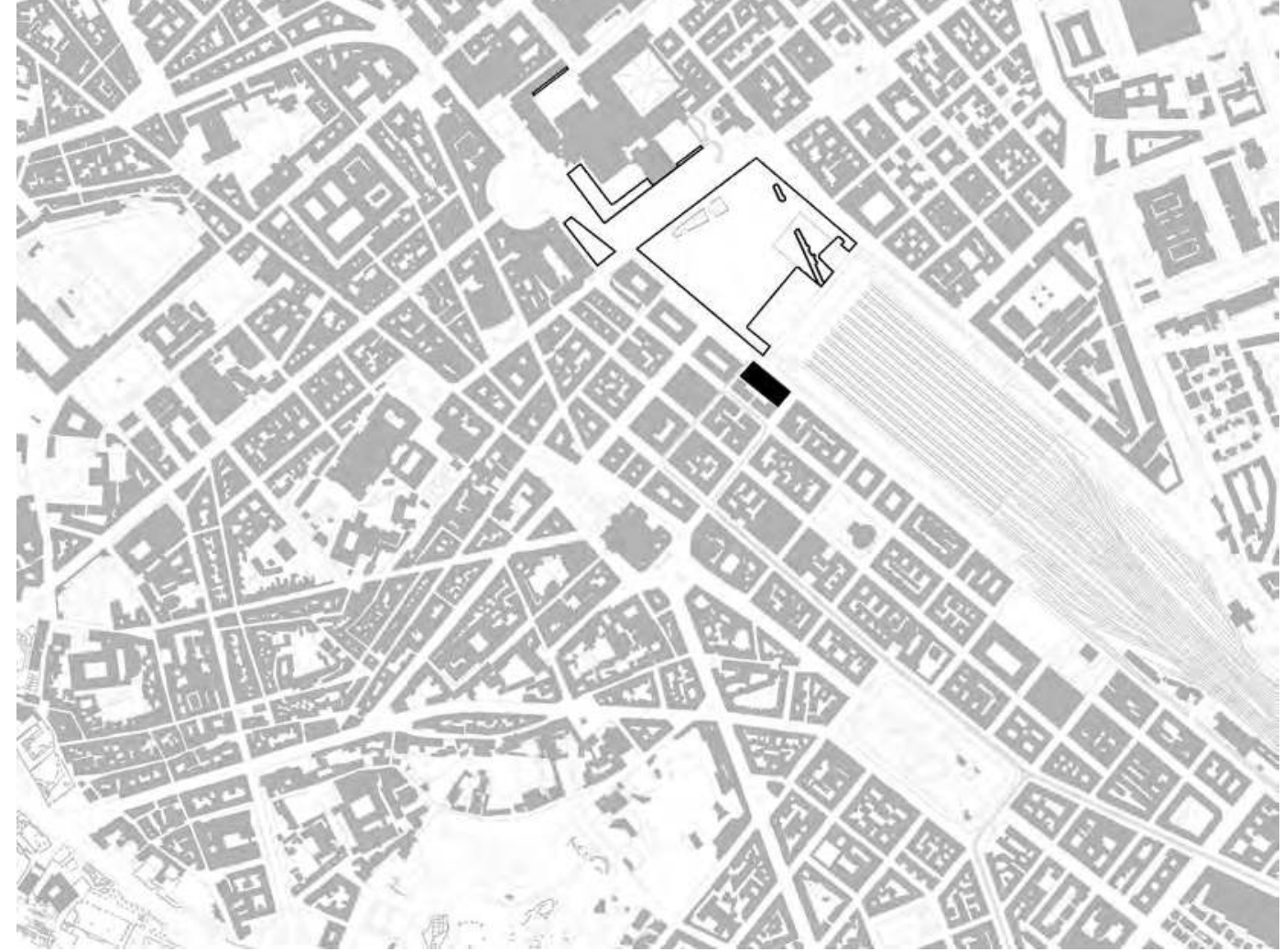

Fig. 3 - L'area della

stazione Termini

a Roma. In nero la

localizzazione dell'in-

tervento. Elaborato

di F. Miele. 
Fig. 4 - Studio delle funzioni per il tipo a corpi autonomi e ipotesi di connessione ipogea con il Forum Termini. Elaborato di S. Leoni.
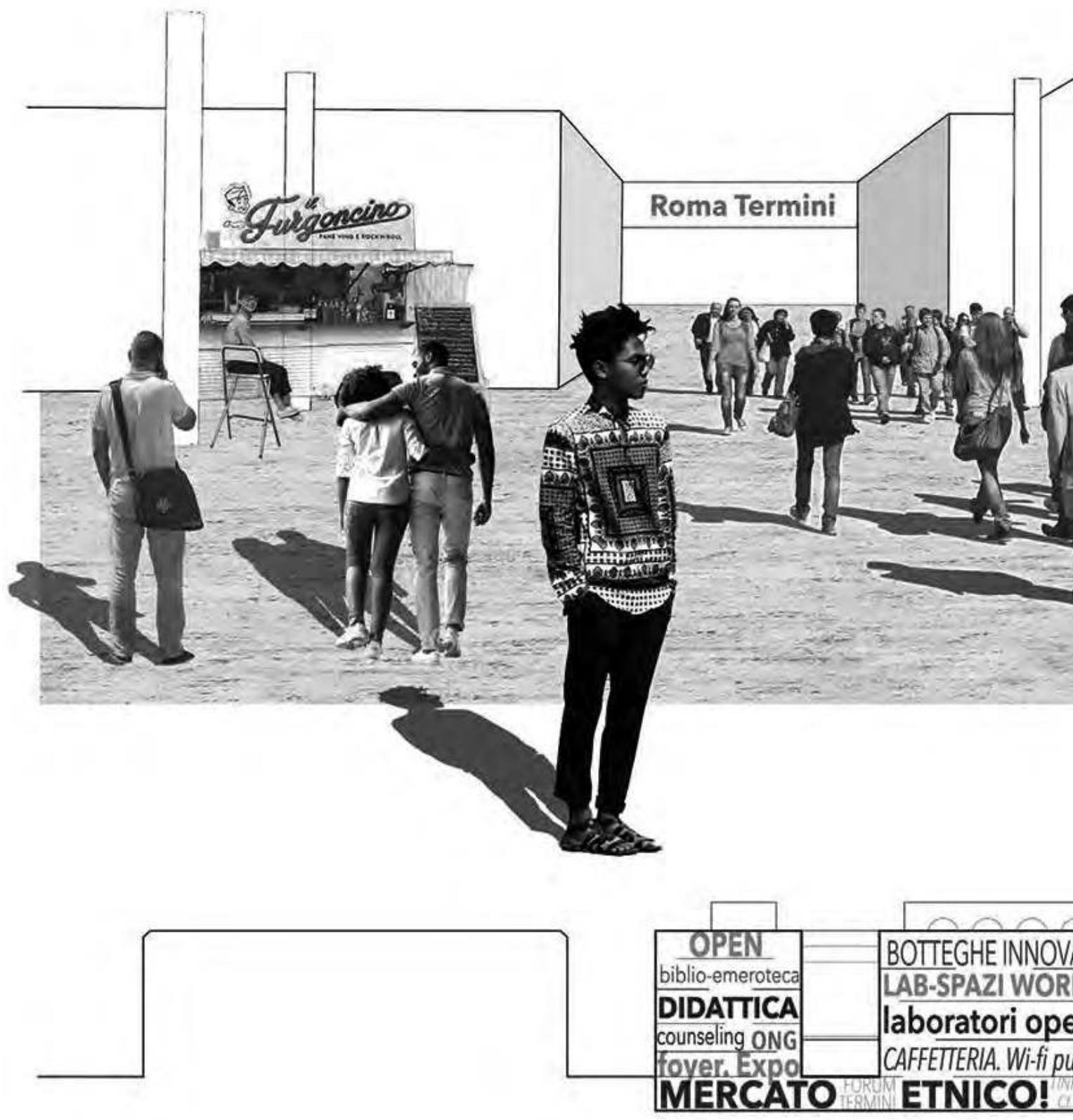

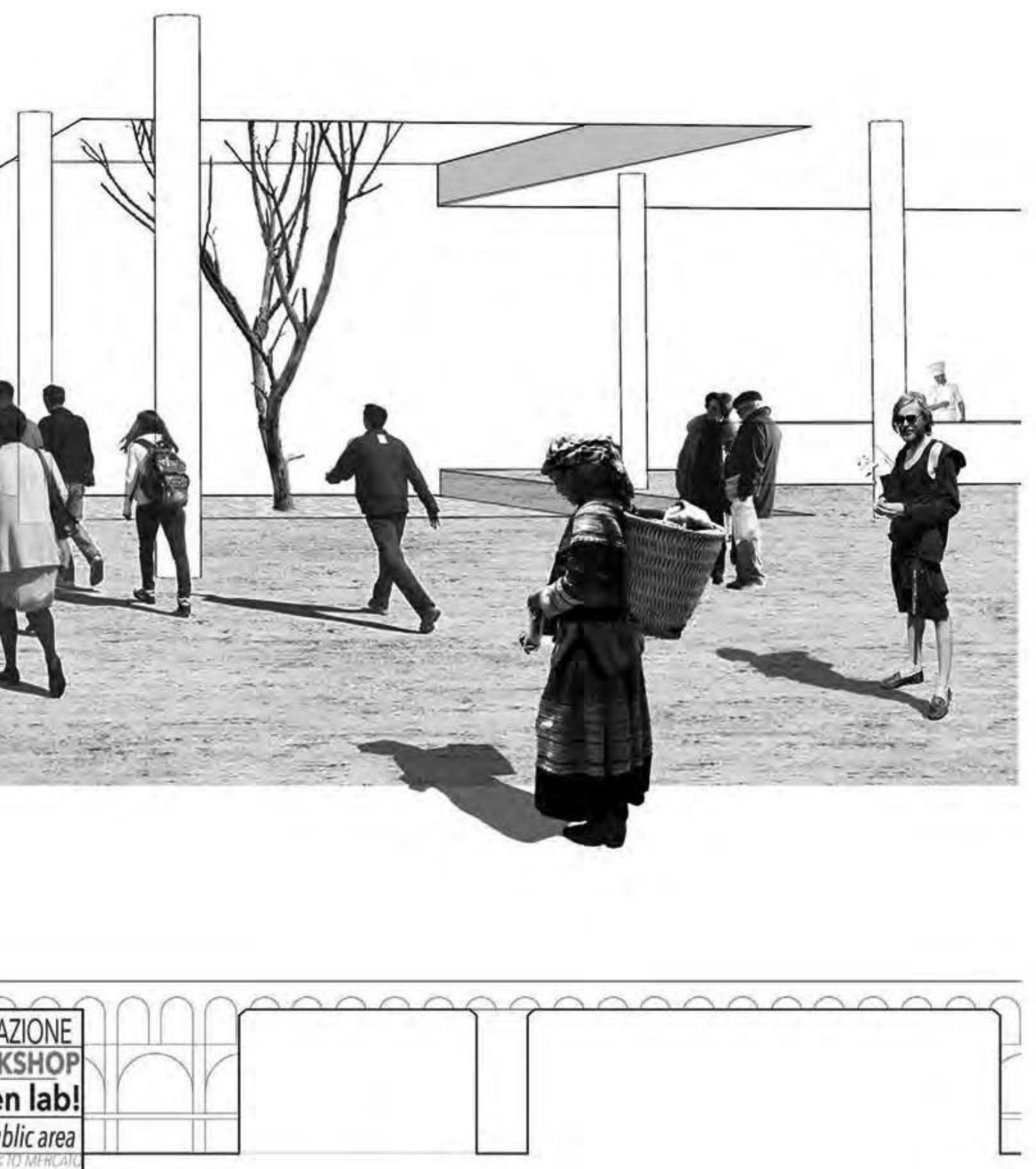

funzionale, ma si tratta piuttosto di studiare un tipo specifico che pone problemi nuovi, quali per esempio la necessità di un doppio registro, tra funzioni molto "delicate" che richiedono un alto grado di privacy (per esempio l'assistenza psicologica) e altre invece aperte al pubblico, quali, per esempio, gli spazi espositivi. Questo genere di edificio, che a noi piace chiamare "Casa delle Culture" per mettere in primo piano il suo carattere plurale, funzionerebbe perciò da un lato da servizio assistenziale, dall'altro da "scambiatore sociale" nel quale attivare un dialogo bidirezionale tra soggetti stranieri (extracomunitari e non) e cittadini nativi. Meno Cité de Refuge, insomma, ma più Cité du Monde.

Con intento esplorativo, tale "condensatore sociale” è stato collocato a lato della stazione Termini, a completamento di un isolato parzialmente demolito negli anni Cinquanta, posto di fronte l'ala mazzoniana e prospiciente l'imbocco della grande galleria passante che collega via Giolitti con via Marsala.

La prossimità della stazione permette di realizzare il collegamento diretto dell'edificio, alla quota $-4,00$, con gli spazi del Forum Termini, consentendo di ubicare nell'interrato alcuni servizi come lavanderie e docce, che vanno considerati parte essenziale della dotazione di questo genere di servizio, poiché spesso i luoghi dove i migranti dormono sono sprovvisti delle strutture minime per ottemperare a una corretta igiene personale.

Negli schemi che sono allo studio, si stanno valutando tre possibili strategie di impianto: l'edificio unitario, organizzato per livelli funzionali, con le aree pubbliche poste alla quota stradale e con le funzioni assi-
Fig. 6 - Studi sul tipo a ponte. Ipotesi di corpo a rampa verso il fronte di Termini. Elaborato di F. Caparello.

\section{Si tratta di studiare un tipo specifico che pone problemi nuovi, quali la necessità di un doppio registro, tra funzioni che richiedono un alto grado di privacy e altre invece aperte al pubblico, quali, per esempio, gli spazi espositivi.}

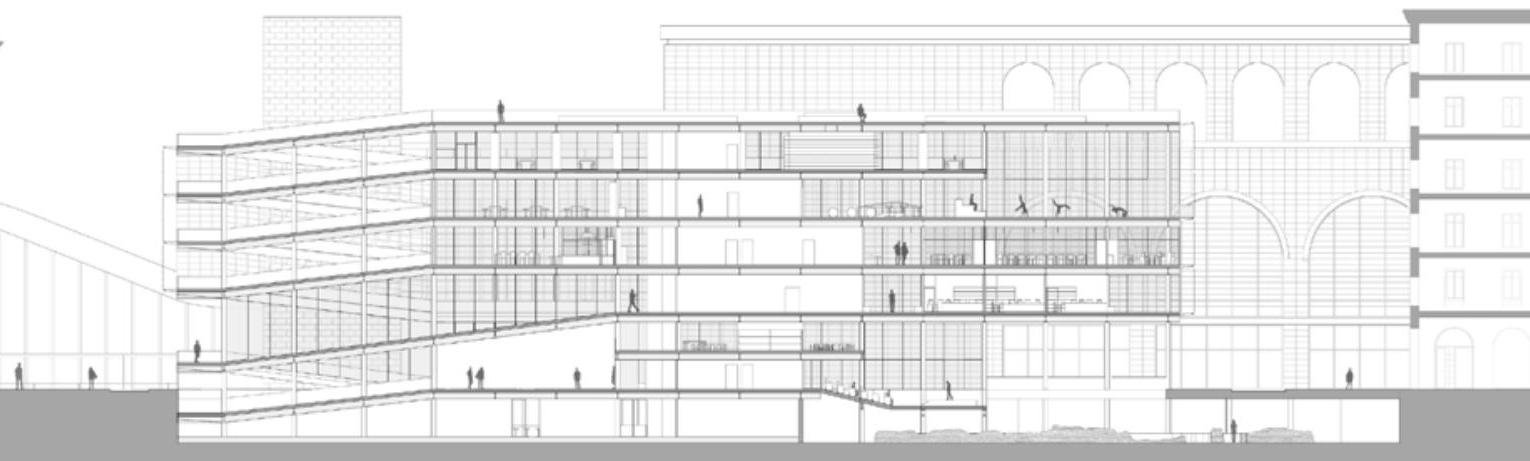




\section{Negli schemi che sono allo studio, si stanno valutando tre possibili strategie di impianto: l'edificio unitario, organizzato per livelli funzionali, il tipo per corpi indipendenti, infine il tipo dell'edificio- ponte.}

stenziali e di supporto collocate in alto o nel basamento interrato; il tipo per corpi indipendenti, connessi solo alla quota interrata o per via di una piastra basamentale; infine il tipo dell'edificio-ponte, con due appoggi a terra distinti, sostanzialmente una variante del precedente, ma connesso in quota tramite un passaggio aereo.

Le ipotesi che qui pubblichiamo sono elaborazioni progettuali condotte dai dottorandi del Dipartimento di Architettura e Progetto che fanno parte dell'unità di ricerca. Queste sviluppano principalmente il paradigma dell'edificio a corpi separati; questo tipo di soluzione si rivela infatti ottimale nel peculiare contesto di Termini, poiché permette di specializzare le parti del complesso, consentendo a uno dei due corpi - quello orientato verso la piazza dei Cinquecento - di caratterizzarsi maggiormente per ospitare le funzioni di scambio attivo con la cittadinanza; inoltre, se sviluppato nella forma dell'edificio-ponte, questo tipo permette anche di definire un fornice urbano posto in diretta connessione con il fornice d'ingresso della galleria della stazione, lungo via Giolitti; infine, tale soluzione d'impianto risponde bene anche all'esigenze di gestione dei flussi, differenziabili attraverso l'innesto di ampie rampe che indirizzano ai servizi culturali, mentre verso via Manin sarebbero rivolti gli accessi riservati, dedicati alle funzioni assistenziali. La scelta di questo genere di soluzioni discende, però, anche da considerazioni del tutto specifiche, inerenti la morfologia del quartiere: qui, dato il tessuto a blocchi dell'Esquilino, gli impianti a doppio corpo ci sembrano assecondare una possibilità di collegamento trasversale verso sud, cioè verso piazza Santa Maria Maggiore, che altrimenti risulterebbe impedita. Non si esclude, tuttavia, che in altre realtà urbane il tipo del contenitore unico polifunzionale possa risultare altrettanto efficace.

Conclusioni. Quale ruolo dell'architettura nell'Europa del nuovo millennio?

In conclusione, ci permettiamo una digressione volta a ricollocare il tema finora trattato in una prospettiva più generale. Se infatti il Movimento Moderno aveva codificato una koเv $\square$ su cui fondare un rinnovato linguaggio architettonico universale, gli anni Cinquanta e Sessanta, a seguito dell'affermazione di discipline 
come la sociologia e l'antropologia (e conseguentemente alle riflessioni indotte dal grande tema della ricostruzione postbellica) hanno generato nuovi paradigmi del fare architettura. Paradigmi che hanno spostato l'accento dagli aspetti linguistici o puro-visibilistici agli aspetti fenomenologici e relazionali: gli spazi dell'abitare collettivo hanno così rappresentato il campo dove sperimentare l'avvento di nuove dimensioni politiche del progetto, che hanno riguardato tanto la ricerca sull'housing quanto gli studi sul tipo del grande condensatore sociale, che è quello cui si fa riferimento nelle proposte qui presentate di "edifici-mondo”. Tali indagini sono state prioritarie fino a tutti gli anni Settanta del Novecento, fintanto che il postmoderno, nel quale siamo ancora pienamente immersi, non ha abbandonato l'impegno politico-sociale a favore della sperimentazione estetico-formale (o della celebrazione tecnologica): in termini generali, l'affermazione dei grandi ideali ha quindi ceduto il passo al disincanto, la condivisione all'individualismo, il futuro al quotidiano; mentre l'attenzione è ripiegata nell'esistenzialismo, avvolgendosi in una cornice più intima e soggettiva, meno pubblica e solidale. D'altronde l'andamento sinusoidale della storia di ogni civiltà, che prevede l'avvicendarsi di fasi ascendenti e discendenti, non ha fatto eccezione per il Novecento, così come era accaduto nelle epoche passate. Ogni opera umana, da sempre, è stata espressione di questo altalenante dualismo. Se per esempio mettiamo a confronto il Discobolo di Mirone del V secolo a.C. con il Pugilatore a riposo di Apollonio di Atene del I secolo a.C., questo contrasto è già ben evidente. La prima scultura, di epoca classica, rappresenta la forza ideale del giovane atleta e l'azione è ritratta nel momento di massima tensione prima del lancio, in pieno contrasto con l'espressione del volto sereno e concentrato. Contrariamente all'atleta vincente, il pugilatore a riposo, di epoca ellenistica, è rappresentato durante una pausa del combattimento, con cicatrici e tagli sul viso, la bocca semiaperta quasi a far percepire il suono di un respiro affannato.

A questa doppia faccia del sentire umano si riferisce José Maria Montaner, che così sintetizza tutte le contraddizioni del secolo breve:

Di fatto questa distanza [...] si può riassumere paragonando due immagini appartenenti paradossalmente allo stesso peri-
L'affermazione dei grandi ideali ha ceduto il passo al disincanto, la condivisione all'individualismo, il futuro al quotidiano; mentre l'attenzione è ripiegata nell'esistenzialismo, avvolgendosi in una cornice più intima e soggettiva, meno pubblica e solidale. 
La difficile

condizione che

stiamo vivendo,

potrà forse

costituire la

premessa per un

cambio di rotta,

come dimostrano le

recenti disposizioni

italiane in tema di

regolarizzazione

dei migranti nel

lavoro dei campi. odo [...]: l'uomo atletico perfetto e muscoloso alto 1 metro e 83, del maschilista Modulor di Le Corbusier ed i personaggi deformi e indigenti che appaiono sperduti sugli sfondi di sabbia e pietra dei primi quadri di Jean Dubuffet (Montaner, 1996).

Né l'uno né l'altro, da soli, basterebbero a designare l'esperienza umana, ma molto da imparare abbiamo da entrambi: dall'uno ereditiamo l'homo faber e dall'altro la fragilità dell'esistere hic et nunc; fragilità che ci riconduce al momento attuale, e alle conseguenze dell'epidemia globale che sta impattando sull'intero pianeta.

La difficile condizione che stiamo vivendo, che sta obbligando l'intera Europa allo sforzo di dimostrarsi qualcosa di più di un'unione solo monetaria, potrà forse costituire la premessa per un cambio di rotta, come dimostrano le recenti disposizioni italiane in tema di regolarizzazione dei migranti nel lavoro dei campi. Se dunque uno scenario virtuoso riuscirà a innescarsi nel prossimo futuro - come accade spesso a seguito delle crisi profonde - il tema dell'accoglienza tornerà ai primi punti dell'agenda europea non più come minaccia ma come opportunità. All'architetto spetterà allora il compito di progettare l'integrazione, nella cornice di città multiculturali nelle quali le persone dovranno avere il diritto di essere distinte, ma anche il diritto di essere indifferenti all'essere distinte dagli altri.

Se dunque riconosciamo alle città europee il compito di favorire tali processi, è necessario ripristinare la dimensione collettiva e politica di una disciplina, quella dell'architetto, che è anche disciplina sociale poiché prima di tutto osserva i fenomeni, e in seguito propone gli spazi del vivere umano, individuale e collettivo; abbracciando, da un lato, l'impegno civico, il ruolo innovativo, l'operoso sguardo verso il futuro del Movimento Moderno (sguardo che ci ha concesso di progettare - e credere - nella costruzione di un futuro migliore), ma tenendo a mente, d'altro lato, ciò che l'uomo di Dubuffet e le cicatrici del Pugilatore ci rammentano: la vulnerabilità del singolo individuo umano. 


\section{Bibliografia}

Agostini, I., Attili, G., Decandia, L., Scandurra, E. (2017), La

città e l'accoglienza, Roma, Manifestolibri.

Bauman, Z. (2016), Per tutti i gusti. La cultura nell'età dei consumi, Roma-Bari, Laterza.

Bauman, Z. (2016), Stranieri alle porte, Roma-Bari, Laterza.

Bauman, Z. (2001), Voglia di comunità, Roma-Bari, Laterza.

Bolzoni, M., Donatiello, D. (2018), Prima accoglienza: obiettivo inclusione, in M. Villa (ed.), Le città globali e la sfida dell'integrazione, Milano, Ledizioni, pp. 61-79.

Borsi L. (ed.) (2017), Dossier Servizi Studi del Senato, XVII legislatura, Immigrazione Elementi Introduttivi, n. 430.

Criconia, A. (ed.) (2019), Una città per tutti. Diritti, spazi, cittadinanza, Roma, Donzelli.

Firouzi, O. (2018), Migranti tra confinamenti mobili e resistenze impreviste: riflessioni da un campo di ricerca, in Studi sulla questione criminale. Nuova serie dei delitti e delle pene [Online]. Available at: https://studiquestionecriminale.wordpress. com/2018/02/15/migranti- tra-confinamenti-mobili-e-resistenze-impreviste-riflessioni-da-un-campo-di-ricerca-di-omid-firouzi-universita-degli-studi-di-padova/ [Accessed: 09 november 2020].

Montaner, J. M. (1996), Dopo il movimento moderno, l'architettura della seconda metà del Novecento, Roma-Bari, Laterza. Pavia, R. (2016) Architettura come accoglienza, in P. Galante (ed.), Migration and the built Environment in the Mediterranean and the Middle East, Napoli, Atti del Convegno internazionale Caumme III/Paumme I.

Urban@it. Centro nazionale studi politiche urbane (ed.) (2016), Rapporto sulle città, metropoli attraverso la crisi, Bologna, il Mulino. 